TOPOLOGICAL ALGEBRAS, THEIR APPLICATIONS,

AND RELATED TOPICS

BANACH CENTER PUBLICATIONS, VOLUME 67

INSTITUTE OF MATHEMATICS

POLISH ACADEMY OF SCIENCES

WARSZAWA 2005

\title{
ELEMENTS OF FINITE CLOSED DESCENT IN BANACH AND FRÉCHET ALGEBRAS
}

\author{
GRAHAM R. ALLAN \\ Department of Pure Mathematics and Mathematical Statistics \\ Centre for Mathematical Sciences, University of Cambridge \\ Wilberforce Road, Cambridge CB3 OWB, U.K. \\ E-mail: g.r.allan@dpmms.cam.ac.uk
}

\begin{abstract}
This paper will give a brief survey of ideas related to 'elements of finite closed descent' in certain kinds of topological algebra.
\end{abstract}

1. Introduction. Let $E$ be a topological vector space (over $\mathbb{R}$ or $\mathbb{C}$ ) and let $T: E \rightarrow E$ be a linear mapping. Consider the decreasing sequence of subspaces,

$$
E \supseteq T E \supseteq T^{2} E \supseteq \ldots
$$

The mapping $T$ is said to have finite closed descent (or FCD) (on $E$ ) iff there is some integer $N \geq 0$ such that $T^{n} E$ is dense in $T^{N} E$ (so that $\overline{T^{n} E}=\overline{T^{N} E}$ ) for every $n \geq N$.

REMARKS. 1. If $T$ is continuous, the FCD-property has the simpler formulation: $T$ has FCD iff there is some $N \geq 0$ such that $\overline{T^{N+1} E}=\overline{T^{N} E}$.

2. The reason that we do not restrict attention to continuous operators is for the statement of the 'Density Lemma' (i.e. Theorem 5) below.

An important special case arises in considering elements of algebras. Thus, let $A$ be an algebra, topologized as (at least) a topological vector space and let $x \in A$; define $L_{x}: A \rightarrow A$ by $L_{x}(y)=x y(y \in A)$. Then the element $x$ is said to have left-FCD iff the operator $L_{x}$ has FCD on $A$.

REMARKs. 1. If $A$ is a topological algebra (i.e. multiplication is, at least, separately continuous) then an element $x$ of $A$ has left-FCD iff there is an integer $N \geq 0$ with $x^{N} \in \overline{x^{N+1} A}$.

2. There is, of course, an analogous notion of 'right-FCD'. If the algebra $A$ is commutative, we just say that ' $x$ has FCD'.

EXAMPLES. 1. If $T$ is either invertible or nilpotent then, trivially, $T$ has FCD.

2000 Mathematics Subject Classification: Primary 46H40, 46M15, 46M40; Secondary 46M20

The paper is in final form and no version of it will be published elsewhere. 
2. Every continuous linear map $T: E \rightarrow E$ with dense range has FCD.

3. Every bounded normal operator $T$ on a Hilbert space has FCD, it being elementary that $\overline{\operatorname{im} T}=\overline{\operatorname{im} T^{2}}$.

4. Let $\Omega$ be a compact Hausdorff space and let $A=C(\Omega)$, the standard commutative Banach algebra of all continuous, complex-valued functions on $\Omega$, with the uniform norm. Then every $f$ in $A$ has FCD, it being elementary that $\overline{A f}=\overline{A f^{2}}$ (because $f$ and $f^{2}$ have the same zero set).

5. Let $V$ be the Volterra algebra, i.e. $V=L^{1}[0,1]$, with multiplication being the 'chopped-off' convolution, denoted by $*$. For each non-zero $f$ in $V$, let $\alpha(f)=\inf \operatorname{supp} f$. Then it is well-known that $\overline{V * f}=V$ if and only if $\alpha(f)=0$, while if $\alpha(f)>0$ then $f$ is properly nilpotent. Thus $V$ is an example of a commutative, radical Banach algebra in which every element has FCD.

6. Let $A=A(\Delta)$ be the usual disc algebra. Then it is elementary to see that, if $f$ is not identically zero, but $f^{-1}(0) \cap$ int $\Delta \neq \varnothing$, then $f$ does not have FCD. But e.g., if $g(z)=1-z$, then $g$ does have FCD.

7. Let $\mathcal{F} \equiv \mathbb{C}[[X]]$ be the algebra of all formal power series in the variable $X$, with complex coefficients; then $\mathcal{F}$ is an example of a commutative Fréchet algebra (see $§ 5$ ), with the topology of coefficientwise convergence. Then the sequence

$$
\mathcal{F} \supset X \mathcal{F} \supset X^{2} \mathcal{F} \supset \ldots
$$

is a properly decreasing sequence of closed ideals of $\mathcal{F}$, so that $X$ is not an element of FCD.

Before going any further, there is an obvious question: why consider the property of finite closed descent?

The first appearance of the condition (though, in the original statement, the property had not been given a name) was in the following theorem (as in the last paragraph, we shall write $\mathcal{F}=\mathbb{C}[[X]])$.

TheOREM 1 (see [1]). Let $R$ be a commutative, unital Banach algebra and let $x \in R$. The following are equivalent:

(i) there is a homomorphism $\theta_{x}: \mathcal{F} \rightarrow R$ with $\theta_{x}(X)=x$;

(ii) $x \in \operatorname{rad} R$ and $x$ has FCD.

REMARKS. 1. In case (i) and (ii) hold, the homomorphism $\theta_{x}$ is injective if and only if $x$ is not nilpotent. From this fact, it is immediate that $\theta_{x}$ cannot be both injective and continuous (with respect to the standard Fréchet topology of $\mathcal{F}$ ).

2. In view of Example 5, if we take $R=V_{+}$, the unitization of the Volterra algebra $V$, and any non-nilpotent element $x$ of $V$, then there is an injective homomorphism $\theta_{x}: \mathcal{F} \rightarrow R$ with $\theta_{x}(X)=x$.

2. Stable elements. It will be useful to introduce a purely algebraic idea, whose relation to the FCD property will appear shortly. The definition to be given relates to homological properties of the inverse-limit functor (see discussion in [3]); but that aspect will not be discussed here. 
Let $V$ be a vector space and let $T \in L(V)$, the algebra of all linear endomorphisms of $V$. We say that $T$ acts stably on $V$ iff for every sequence $\left(v_{n}\right)$ of elements of $V$, there is a sequence $\left(x_{n}\right)$ in $V$, such that

$$
x_{n}=T\left(x_{n+1}\right)+v_{n} \quad(n \geq 1) .
$$

ExAmPLES. 1. If $T(V)=V$, then evidently $T$ acts stably.

2. If $T$ is nilpotent, then it is also clear that $T$ acts stably, for, given $\left(v_{n}\right)$, we may take $x_{n}=v_{n}+T v_{n+1}+T^{2} v_{n+2}+\cdots$.

Non-trivial examples will appear later.

As with the definitions of FCD, an important special case arises when $T$ is taken to be multiplication by an element of an algebra.

Let $A$ be an algebra and let $x \in A$. Then $x$ is said to be a left-stable element iff $L_{x}$ acts stably on $A$.

REMARK. There is, of course, an analogous notion of 'right-stable element'. If the algebra $A$ is commutative, we just say that ' $x$ is stable'.

There are two simple lemmas relating to stable action, which will be used later. Their proofs could be exercises for an interested reader.

Lemma 2. Let $V$ be a vector space and $T \in L(V)$. The following are equivalent:

(i) $T$ acts stably on $V$;

(ii) for every $k \geq 1, T^{k}$ acts stably on $V$;

(iii) for some $k \geq 1, T^{k}$ acts stably on $V$.

If $V$ is a vector space, $T \in L(V)$ and if $U$ is a $T$-invariant subspace of $V$, then $T$ naturally induces linear endomorphisms of $U$ and of $V / U$; we shall simply say that $T$ acts on $U$ and on $V / U$.

Lemma 3. Let $V$ be a vector space, let $T \in L(V)$ and let $U$ be a $T$-invariant subspace of $V$. Then:

(i) if $T$ acts stably on $V$, then it also acts stably on $V / U$;

(ii) if $T$ acts stably on both $U$ and on $V / U$, then $T$ acts stably on $V$.

The definition of stable action is a special case of the definition of a so-called stable inverse-limit sequence; it will be helpful to make a few comments about this more general case. An inverse-limit sequence of vector spaces and linear mappings, is a sequence of the following type:

$$
\mathcal{V}: \quad V_{1} \stackrel{T_{1}}{\longleftarrow} V_{2} \stackrel{T_{2}}{\longleftarrow} V_{3} \stackrel{T_{3}}{\longleftarrow} \cdots,
$$

each $V_{n}$ being a vector space, and each $T_{n}: V_{n+1} \rightarrow V_{n}$ a linear mapping (the symbol $\mathcal{V}$ just gives a name to the sequence).

The sequence $\mathcal{V}$ is called stable iff, for every sequence $\left(v_{n}\right)$, where $v_{n} \in V_{n}(n \geq 1)$, there are elements $x_{n} \in V_{n}$ such that $x_{n}=T_{n}\left(x_{n+1}\right)+v_{n}(n \geq 1)$.

In terms of this more general definition, it is then clear that the linear mapping $T: V \rightarrow V$ acts stably on $V$ iff the sequence

$$
V \stackrel{T}{\longleftarrow} V \stackrel{T}{\longleftarrow} V \stackrel{T}{\longleftarrow} \cdots,
$$

formed by iterating the action of $T$, is a stable inverse-limit sequence. 
The main reason for introducing the more general stability definition is to be able to use another special case. Let $V$ be a vector space and let

$$
\mathcal{V}: \quad V_{1} \supseteq V_{2} \supseteq V_{3} \supseteq \ldots
$$

be a decreasing sequence of subspaces of $V$. Then the sequence $\mathcal{V}$ is called a stable sequence of subspaces of $V$ provided that it is stable regarded as an inverse-limit sequence

$$
\mathcal{V}: \quad V_{1} \stackrel{j_{1}}{\longleftarrow} V_{2} \stackrel{j_{2}}{\longleftarrow} V_{3} \stackrel{j_{3}}{\longleftarrow} \cdots,
$$

in which each $j_{n}$ is an inclusion mapping.

Explicitly, the decreasing sequence $\left(V_{n}\right)$ of subspaces of $V$ is stable iff for every choice of $v_{n} \in V_{n}(n \geq 1)$, there are $u_{n} \in V_{n}$ such that $u_{n}=u_{n+1}+v_{n}(n \geq 1)$.

There is the following very simple lemma that connects the two special cases.

Lemma 4. Let $V$ be a vector space and let $T \in L(V)$. If $T$ acts stably on $V$, then $\left(T^{n} V\right)_{n \geq 1}$ is a stable sequence of subspaces of $V$.

Proof. Let $T$ act stably on $V$ and let $v_{n} \in T^{n} V(n \geq 1)$. Then, for each $n$ we have $v_{n}=T^{n}\left(y_{n}\right)$ for some $y_{n} \in V$.

By the stable action of $T$, there is then a sequence $\left(x_{n}\right)$ in $V$ such that $x_{n}=T x_{n+1}+y_{n}$ for every $n$. Define $u_{n}=T^{n}\left(x_{n}\right)(n \geq 1)$ and we see at once that, for each $n$,

$$
u_{n}=T^{n}\left(x_{n}\right)=T^{n+1}\left(x_{n+1}\right)+T^{n}\left(y_{n}\right)=u_{n+1}+v_{n} .
$$

It follows that $\left(T^{n} V\right)$ is a stable sequence of subspaces of $V$.

That the algebraic notion of stability is related to the topological-algebraic notion of FCD begins to emerge with the following result.

TheOREM 5 (Density Lemma). Let $V$ be a vector space and let $\mathcal{V}=\left(V_{n}\right)_{n \geq 1}$ be a stable sequence of subspaces of $V$. Let $p$ be a seminorm on $V$. Then there is an integer $N \geq 1$ such that $V_{n}$ is $p$-dense in $V_{N}$ for every $n \geq N$.

Using Lemma 4, there is then the following immediate corollary:

Corollary 6. Let $V$ be a vector space, let $T \in L(V)$ and suppose that $T$ acts stably on $V$. Then, for any seminorm $p$ on $V, T$ has FCD with respect to $p$.

To get implications in the other direction, from FCD properties to stability, we begin by recalling the abstract Mittag-Leffler theorem (which is a generalized Baire-Category result).

ThEOREM 7 (Mittag-Leffler). Let

$$
X_{1} \stackrel{f_{1}}{\longleftarrow} X_{2} \stackrel{f_{2}}{\longleftarrow} X_{3} \stackrel{f_{3}}{\longleftarrow} \cdots
$$

be a sequence of (non-empty) complete metrizable topological spaces and continuous mappings, such that, for all $n \geq 1, f_{n}\left(X_{n+1}\right)$ is dense in $X_{n}$. Then there is a dense subset, say $D$, of $X_{1}$ such that, for every $d \in D$, there is a sequence $\left(x_{n}\right)_{n \geq 1}$, with $x_{n} \in X_{n}$, $x_{1}=d$ and $x_{n}=f_{n}\left(x_{n+1}\right)$ for all $n \geq 1$.

Corollary 8. Let $V$ be a complete-metrizable topological vector space, and let $T: V \rightarrow$ $V$ be a continuous linear mapping with $\overline{T(V)}=V$. Then $T$ acts stably on $V$. 
Proof. Let $\left(v_{n}\right)$ be a sequence in $V$ and, for each $n \geq 1$ define $f_{n}: V \rightarrow V$ by $f_{n}(x)=$ $T(x)+v_{n}(x \in V)$. Evidently $\overline{f_{n}(V)}=V$ for all $n$, so that the sequence

$$
V \stackrel{f_{1}}{\longleftarrow} V \stackrel{f_{2}}{\longleftarrow} V \stackrel{f_{3}}{\longleftarrow} \ldots
$$

satisfies the hypothesis of Theorem 7 . Hence there is a sequence $\left(x_{n}\right)$ in $V$ such that $x_{n}=f_{n}\left(x_{n+1}\right)=T\left(x_{n+1}\right)+v_{n}$, for all $n$. This completes the proof.

Corollary 9. Let $V$ be a complete-metrizable topological vector space, and let $T: V \rightarrow$ $V$ be a continuous linear mapping that has FCD. Then $T$ acts stably on $V$.

Proof. By hypothesis, there is an integer $N \geq 1$ such that $\overline{T^{n} V}=\overline{T^{N} V}$ for all $n \geq N$.

Let $S=T^{N}$ and let $Y=\overline{S(V)}$; then $Y$ is a closed, $S$-invariant subspace and

$$
Y \supseteq \overline{S(Y)} \supseteq \overline{T^{N}\left(T^{N} V\right)}=\overline{T^{N}(V)}=Y .
$$

By Corollary 8, $S$ acts stably on $Y$. Also, since $S(V) \subseteq Y, S$ acts on $V / Y$ as the zero-operator, which action is trivially stable.

By Lemma 3 (ii), $S$ acts stably on $V$ and so, by Lemma 2, $T$ acts stably on $V$.

TheOREM 10. Let $E$ be a Banach space and let $T$ be a bounded linear operator on $E$. Then $T$ acts stably if and only if it has FCD.

Proof. This follows immediately from Corollaries 6 and 9.

The special case for Banach-algebra elements now follows at once.

Corollary 11. Let $A$ be a Banach algebra and let $x \in A$. Then $x$ is left-stable if and only if it has left-FCD.

Corollary 12. (i) Let $E$ be a Banach space and let $T \in \mathcal{B}(E)$ have $F C D$. Let $p$ be an arbitrary seminorm on $E$; then $T$ also has FCD with respect to $p$.

(ii) Let $A$ be a Banach algebra and let $x$ be an element of $A$ that has left-FCD. Then $x$ also has left-FCD with respect to an arbitrary (linear-space) seminorm on $A$.

Proof. (i) By Theorem 10, $T$ acts stably on $E$. But then, by the corollary to the Density Lemma (Corollary 6 ), $T$ acts stably with respect to $p$.

(ii) This is a special case of (i).

The next corollary gives the somewhat surprising result that the property of having FCD is preserved by an arbitrary (i.e. not necessarily continuous) homomorphism of Banach algebras.

Corollary 13. Let $A, B$ be Banach algebras and let $F: A \rightarrow B$ be a homomorphism (not necessarily continuous). Let the element $x$ of $A$ have left-FCD; then $F(x)$ has left-FCD in $B$.

Proof. Define a seminorm $p$ on $A$ by $p(a)=\|F(a)\|_{B}$. By Corollary 12(ii), $x$ has left-FCD with respect to $p$.

Thus there is an integer $N \geq 0$ and a sequence $\left(a_{k}\right)_{k \geq 1}$ in $A$, such that

$$
0=\lim _{k \rightarrow \infty} p\left(x^{N}-x^{N+1} a_{k}\right)=\lim _{k \rightarrow \infty}\left\|F(x)^{N}-F(x)^{N+1} F\left(a_{k}\right)\right\|_{B} .
$$

Thus $F(x)$ has left-FCD in $B$. 
The stability viewpoint is closely connected to the embedding of formal power series in commutative algebras. Thus, let $A$ be a commutative algebra with identity and let $x \in A$. We define two ideals that are associated with the element $x$; the first is simply $I(x)=\bigcap_{n \geq 1} A x^{n}$. The second, $I_{0}(x)$, is defined by saying that $a \in I_{0}(x)$ if and only if there is a sequence $\left(a_{n}\right)_{n \geq 0}$ in $A$ such that $a_{0}=a$ and $a_{n}=a_{n+1} x$ for all $n \geq 0$. Evidently $I_{0}(x) \subseteq I(x)$, and it is simple to see that $I_{0}(x)=I(x)$ if and only if $L_{x}(I(x))=I(x)$. (As before, $L_{x}$ is the operation of (left) multiplication by $x$.) This property holds, in particular, if $A$ is a commutative topological algebra and $x$ is an element of $A$ having FCD. (It also holds if $A$ is a commutative Fréchet algebra and the element $x$ has the weaker property of 'locally finite closed descent'; the definition is in §4.) In a purely algebraic context, I do not know whether, for a stable element of a commutative algebra, it is always true that $I_{0}(x)=I(x)$; probably it is false, but I do not know a counter-example.

Let $A$ be a commutative algebra, let $x \in A$ and let $\pi_{x}: A \rightarrow A / I_{0}$ be the quotient mapping.

TheOREM 14. Let $A$ be a commutative, unital algebra and let $x \in A$. Then $x$ is stable if and only if there is a homomorphism $\Psi_{x}: A[[X]] \rightarrow A / I_{0}(x)$ such that both $\Psi_{x}(X)=$ $\pi_{x}(x)$ and also $\Psi_{x}(a)=\pi_{x}(a)$ for every $a \in A$.

In this case, the homomorphism $\Psi_{x}$ is uniquely determined, $\operatorname{im} \Psi_{x}=A / I_{0}(x)$ and $\operatorname{ker} \Psi_{x}=(X-x) A[[X]]$.

Proof. See [4] Theorem 3.4 (slightly improved through Lemma 3.1 of that paper).

3. Further significance of the FCD property. The FCD condition has also arisen in:

(i) work of Dales and Esterle on the Kaplansky problem (1976 et seq; see [6], [7], [8], [9], [10] and other references given in [5]);

(ii) work of Marc Thomas on the Singer-Wermer conjecture (see [11] and [12]).

As an example of (i):

TheOrem 15 (Esterle). Let $R$ be a commutative, unital Banach algebra. The following are equivalent:

(i) $\operatorname{rad} R$ contains a non-nilpotent element of FCD;

(ii) there is an injective homomorphism of $\mathbb{C}[[X]]$ into $R$;

(iii) (with $\mathrm{CH}$ ) there is a discontinuous homomorphism $C(\Omega) \rightarrow R$ (for some/every infinite, compact $\Omega$ );

(iv) (with $\mathrm{CH}$ ) for every commutative, complex algebra $D$ which is an integral domain of cardinality $2^{\aleph_{0}}$ and which has at least one character, there is an injective homomorphism from $D$ into $R$.

REmarks. 1. The equivalence of (i) and (ii) repeats Theorem 1; (iv) includes (i), except that (iv) needs $\mathrm{CH}$ (or, at least, some set-theoretic axiom beyond ZFC). The $\mathrm{CH}$ is used in the implications (i) $\Rightarrow$ (iii), (i) $\Rightarrow$ (iv).

2. The most convenient place to study this theorem, and many related matters is [5] (whose Theorem 5.7.30 includes our Theorem 15). 
4. Fréchet algebras and FCD. There has already been reference at this meeting to Marc Thomas's work on Fréchet algebras, where FCD-type concepts also arise. We shall look quickly at the appropriate modifications when going from Banach to more general complete metrizable topological algebras, primarily Fréchet algebras.

Terminology. We follow the terminology of the Polish school, by using the term $B_{0}$-algebra for a complete metrizable, locally convex topological algebra.

A Fréchet algebra is a multiplicatively convex $B_{0}$-algebra (whose topology may, therefore, be defined by an increasing sequence of submultiplicative seminorms).

First, consider stability: this concept is purely algebraic, and no change is needed. It is easy to see that part of Corollary 11 extends even to $B_{0}$-algebras:

Theorem 16. Let $A$ be a $B_{0}$-algebra and let $x \in A$. If $x$ has (left-)FCD, then $x$ is (left-)stable.

Proof. This is immediate from Corollary 9.

But, in contrast to the Banach-algebra case, the converse is false, even for commutative Fréchet algebras

ExAmple. Let $\mathcal{F}=\mathbb{C}[[X]]$; then $X$ is a stable element of $\mathcal{F}$ but does not have FCD.

However, if $x$ is a (left-)stable element of any algebra $A$, the Density Lemma (Theorem 5) shows that $x$ does have (left-)FCD relative to any single seminorm on $A$. This hints at the 'correct' notion (which will just be given for algebras).

Definition. Let $A$ be a $B_{0}$-algebra and let $x \in A$. Say that $x$ has (left-) locally finite closed descent (LFCD) if and only if, for every continuous seminorm $p$ on $A, x$ has (left-)FCD relative to $p$.

REMARKS. 1. If $\left(p_{n}\right)_{n \geq 1}$ is an increasing sequence of continuous seminorms on $A$ that define its topology, then the condition that $x$ have (left-)LFCD is equivalent to $x$ having (left-)FCD with respect to each $p_{n}$. For, if $p$ is any continuous seminorm on $A$, there is some $k \geq 1$ and $C>0$ with $p \leq C p_{k}$. Then there is an integer $N(k)$ such that, with a hopefully obvious notation, for every $j \geq N(k)$,

$$
x^{N(k)} A \subseteq{\overline{x^{j} A}}^{p_{k}} \subseteq{\overline{x^{j} A}}^{p} .
$$

2. If $A$ is a Fréchet algebra then, in (1), we may take each $p_{n}$ to be submultiplicative. So the condition then reduces to the requirement that, for each $k \geq 1$, there is an integer $N(k) \geq 0$ such that

$$
x^{N(k)} \in{\overline{x^{N(k)+1} A}}^{p_{k}} .
$$

3. If $A$ is a Banach algebra, then $x$ has (left-)LFCD if and only if it has (left-)FCD. Theorem 17. Let $A$ be a $B_{0}$-algebra and let $x \in A$. Then $x$ is (left-)stable if and only if $x$ has (left-)LFCD.

Corollary 18. Let $A$ be a $B_{0}$-algebra, let $x \in A$ and let $x$ have (left-)LFCD. Let $p$ be an arbitrary seminorm on $A$. Then $x$ has (left-)FCD relative to $p$. 
It now becomes important to realize that, for a general Fréchet algebra, there is a possibility that does not arise for Banach algebras - namely a property that is intermediate between nilpotence and topological nilpotence. For simplicity, we will now restrict to commutative Fréchet algebras.

Thus, let $R$ be a commutative Fréchet algebra and let $x \in R$; we say that $x$ is locally nilpotent iff, for each continuous seminorm $p$ on $R$, there is an integer $k(p) \geq 1$ with $p\left(x^{k(p)}\right)=0$.

REMARKS. (i) As for the FCD condition, if $\left(p_{n}\right)_{n \geq 1}$ is a defining sequence of seminorms for the topology of $R$, then $x$ is locally nilpotent iff, for all $n \geq 1$, there is an integer $k_{n} \geq 1$ with $p_{n}\left(x^{k_{n}}\right)=0$. (If $p_{n}$ is submultiplicative then clearly $p_{n}\left(x^{k}\right)=0$ for all $k \geq k_{n}$.)

(ii) If $x$ is locally nilpotent then $x$ has LFCD and also $x$ is topologically nilpotent (i.e. Sp $x=\{0\})$.

(iii) If $R$ is a Banach algebra, then $x \in R$ is locally nilpotent iff $x$ is nilpotent.

(iv) In the Fréchet algebra $\mathcal{F}=\mathbb{C}[[X]]$, the element $X$ is locally nilpotent but is not nilpotent.

We have a satisfactory generalization of Theorem 1.

TheOrem 19. Let $R$ be a commutative, unital Fréchet algebra and let $x \in R$. The following are equivalent:

(i) there is a homomorphism $\theta_{x}: \mathcal{F} \rightarrow R$ with $\theta_{x}(X)=x$;

(ii) $x \in \operatorname{rad} R$ and $x$ has LFCD.

In case (i) and (ii) hold then:

(a) $\theta_{x}$ is injective iff $x$ is not nilpotent;

(b) $\theta_{x}$ is a continuous, injective homomorphism iff $x$ is locally nilpotent and nonnilpotent (in which case, also, $\theta_{x}$ has closed range, is a homeomorphism onto its range, and is uniquely defined by the requirement that $\left.\theta_{x}(X)=x\right)$.

Proof. See [2] Theorems 7 and 5.

(v) Let $R$ be a commutative Banach algebra and let $x \in R$ have FCD; thus there is an integer $N \geq 1$ such that $\overline{R x^{n}}=\overline{R x^{N}}$ for all $n \geq N$. An application of the Mittag-Leffler theorem then shows that also $\overline{I(x)}=\overline{R x^{N}}$. It follows that $x$ is nilpotent iff $I(x)=0$.

It may then be deduced that, if $R$ is a commutative Fréchet algebra and if $x \in R$ has LFCD, then $x$ is locally nilpotent iff $I(x)=0$.

Lemma 20. Let $A, B$ be commutative Fréchet algebras, $\theta: A \rightarrow B$ a homomorphism (not necessarily continuous). Then:

(i) if $x \in A$ has LFCD, then $\theta(x)$ has LFCD in $B$;

(ii) if $\theta$ is injective, and if $x \in A$ has LFCD, but is not locally nilpotent, then also $\theta(x)$ is not locally nilpotent.

Corollary 21. Let $\Omega$ be a compact Hausdorff space, $R$ a commutative Fréchet algebra and let $\theta: C(\Omega) \rightarrow R$ be an injective homomrphism. Then every element of $\operatorname{im} \theta \backslash\{0\}$ has LFCD, but is not locally nilpotent. 
TheOrem 22 (M. K. Kopp (2002)). Let $\Omega$ be an infinite, compact Hausdorff space and let $R$ be a commutative, unital Fréchet algebra, whose radical contains a non-locally nilpotent element of LFCD. Then (subject to $C H$ ) there is a discontinuous homomorphism $C(\Omega) \rightarrow R$.

\section{References}

[1] G. R. Allan, Embedding the algebra of formal power series in a Banach algebra, Proc. London Math. Soc. (3) 25 (1972), 329-340.

[2] - Fréchet algebras and formal power series, Studia Math. 119 (1996), 271-288.

[3] - Stable inverse-limit sequences, with application to Fréchet algebras, Studia Math. 121 (1996), 277-308.

[4] - Stable elements of Banach and Fréchet algebras, Studia Math. 129 (1998), 67-96.

[5] H. G. Dales, Banach Algebras and Automatic Continuity, London Math. Soc. Monographs, 24 (OUP, 2000).

[6] -, A discontinuous homomorphism from $C(X)$, Amer. J. Math. 101 (1979), 647-734.

[7] H. G. Dales and J. Esterle, Discontinuous homomorphisms from $C(X)$, Bull. Amer. Math. Soc. 83 (1977), 257-259.

[8] J. Esterle, Sur l'existence d'un homomorphisme discontinu de $C(K)$, Proc. London Math. Soc. (3) 36 (1978), 46-58.

[9] - Homomorphismes discontinus des algèbres de Banach commutatives séparables, Studia Math. 66 (1979), 119-141.

[10] - Universal properties of some commutative radical Banach algebras, J. Reine Angew. Math. 321 (1981), 1-24.

[11] M. P. Thomas, The image of a derivation is contained in the radical, Ann. of Math. 128 (2) (1988), 435-460.

[12] - Local power series quotients of commutative Banach and Fréchet algebras, preprint (2001). 